\title{
Web Performance Analysis: An Empirical Analysis of E-Commerce Sites in Bangladesh
}

\author{
Md. Tutul Hossain, Rakib Hassan, Mahfida Amjad \\ Department of Computer Science \& Engineering, Stamford University Bangladesh, Dhaka, Bangladesh \\ E-mail: \{tutulhossain.cse, tipusultan9t7a\}@gmail.com, mahfidaamjad@stamforduniversity.edu.bd
}

\section{Md. Abdur Rahman}

Centre for Advanced Research in Sciences (CARS), University of Dhaka, Dhaka, Bangladesh

E-mail: mukul.arahman@gmail.com

Received: 20 April 2021; Accepted: 06 June 2021; Published: 08 August 2021

\begin{abstract}
Performance testing of e-commerce site is important for upcoming improvement and making better user experience which is performed by several web performance testing tools available on online platform. There are several tools user can use to scan their site for performance testing. This paper presents a web based application to collect and compare performance parameters with results automatically by applying WebpageTest, PageSpeed Insights and GTmetrix tools. For doing the test comparison nine parameters are considered and these are Load Time, First Byte, Start Render, First Contentful Paint, Speed Index, Largest Contentful Paint, Cumulative Layout Shift, Total Blocking Time and Time to Interactive parameters. The framework is developed with PHP, MySQL, CSS and HTML, where user will provide intended site's url to test performance. This paper presents the performance of ten e-commerce sites of Bangladesh. Among the three tools WebpageTest and Gtmetrix can collect the reports of all the parameters. 1.62 (site7), 3.25 (site4) and 1.89 (site7) seconds are reported as lowest value for tools WebPageTest, PageSpeed Insight and Gtmetrix respectively. The average results of three tools is measured where, the minimum value is shown as 0.03 seconds for 'total blocking time' by site7. And maximum value is shown as 17.78 seconds for 'load time' parameter recorded by site 10 .
\end{abstract}

Index Terms: Web performance testing tools, scalability, speed, stability, load time.

\section{Introduction}

E-commerce sites handles large number of users concurrently. As the number of users increases day-by-day, the performance of the sites plays significant role to keep the user satisfied and thus keep site trends in a good shape. Performance testing is a non-functional requirement which check the system parameters in terms of responsiveness and stability beneath varied workloads. This testing is performed by different scanners available on the online-platforms. These tools live the standard attributes of the system, like quantifiable, dependableness, resource usage, etc.

There are several advantages of performance testing tools like (i) validate the basic options of the system, (ii) measure the speed, accuracy and stability of system, (iii) determine discrepancies and resolve problems, (iv) improve optimization and load capability, etc. Performance testing also examines the sites behavior on multiple devices.

There are several existing works related to website performance analysis such as paper [1] discussed performance testing as well other testing methods to handle the website development challenges. Comparison among three automated user acceptance testing tools for web application has been described in [2]. The work [3] presents a load based testing tool which allows the performance analysis of web applications by means of scalability. The research [6] presented, a model based performance testing tool that measures the performance on web applications and services using the measurement technique. Whereas, the work [7] described an approach to automatically analyzes the execution logs of a load test for performance problems. The new automation testing tool selenium web driver has been described in [8]. The paper [9] investigates open source web service testing tools for lack of parameters such as response time, number of data processed in terms of bytes and throughput. The paper [10] presents a comparison made between selenium and other tools to find the best tool and on the basis of the results; a case study is presented to explore the performance of the selenium. Even though several performance analysis research exists in the literature and discussed different web scanner tools like PageSpeed Insights, Webpage test and Gtmetrix. However, an automatic web framework is essential to collect web performance data using different scanners and compare the results. Which will remove users hassle and difficulties in terms of using different tools for the same website. 
To this end, a web based framework is presented in this research to collect web performance records, analyze, compare and finally store for future usage. Three reputed web performance analyzer has been incorporated to collect and compare the e-commerce site data. This framework will show how a website can behave and respond during various situations. Thus it will help developers to test the speed and stability of their website.

The framework takes the urls as input and produce the test results of each website using three different tools which are (i) WebpageTest (ii) PageSpeed Insights and (iii) GTmetrix. The results are stored to the database for further use and analysis. Also, the system will create a graphical comparison representation among all the sites based on considered performance parameters such as Load time, First Byte, Start Render, etc. using the stored results. In addition, the system has provision to save the comparison results by the registered users.

The proposed system contains two modules named as frontend and backend module which are developed with PHP, MySQL, CSS, HTML. The user will interact with frontend module to collection website performance records after getting registered. A user can scan web performance records of web applications by providing the respective website url. The framework will scan the given sites automatically using the mentioned three tools.

According to the result analysis among ten web applications, the lowest and highest average performance score is 1.62 (site7) and 10.99 (site8) respectively shown by WebpageTest tool. The PageSpeed Insight tool records 3.25 (site4) and 10.12 (site2) as minimum and maximum seconds respectively by PageSpeed Insight tool. However, the tool reports 0.00 second for 'cumulative layout shift' by site8, which indicates either the tool could not detect any record or the site consumes 0 second for this parameter. Where, the site 2 records 24.10 seconds for 'time to interactive' parameter, so this site will take more time to be interactive compared to other experimented sites. The third tool, GTmetrix reports 1.89 (site7) and 9.56 (site8) seconds as lowest and highest average performance respectively.

Finally, the average records of these three tools are measured where, the lowest value is shown as 0.03 seconds for 'total blocking time' by site7. And highest value is 17.78 seconds for 'load time' parameter recorded by site 10 . Three tools' bottommost and uppermost average performance is 2.34 and 9.53 seconds for site 7 and site 8 respectively.

The main contribution of this performance testing framework is to collect performance data automatically and generate graphs depend on testing performance data. Also, user can compare and save two or more site results at a time with a single click.

The rest of this paper is organized as follows; Section 2 discusses the related work. Section 3 is about empirical data collection. Section 4 illustrates the data analysis. Finally, section 5 concludes this paper with future research direction.

\section{Related Works}

There are some existing works related to website performance analysis. There are different types of testing like Functional Testing, Usability Testing, Performance testing, Security Testing, Interface Testing and Compatibility Testing methods discussed in [1] which will help in handling challenges during the website development. Comparison among three automated user acceptance testing tools for web application has been described in [2]. The evaluation is based on the usability criteria of the tools. The three tools evaluated are Acceptance Test Driven Development. Three proposed tools are Test Complete, Selenium Web driver and Watir Web driver. These three tools undergo usability criteria testing that include efficiency, effectiveness, satisfaction and error. From the evaluation, Watir Web driver has been proven as the best testing tool based on usability criteria of user acceptance testing.

The work [3] presents a Web Application Load based Testing tool which is a set of tools that allows the performance analysis of web applications by means of scalability. It is based on workload characterization generated from information extracted from log files. The workload is generated by using customer behavior model graphs. The workload used to evaluate the web application under test which is the representative of the real traffic that the web application has to serve. In [6], they present a model based performance testing tool that measures the performance on web applications and services using the measurement technique. The tool uses models to generate workload which is in real-time and it measures different performance indicators. The models are defined using probabilistic timed automata. It describes how load is generated from the models and the features of the tool.

An approach which automatically analyzes the execution logs of a load test for performance problems has been described in [7]. They first derive the system's performance baseline from previous runs. Then they perform an in-depth performance comparison against the derived performance baseline. Case studies show that their approach produces few false alarms with a precision of $77 \%$. A new automation testing tool Selenium web driver has been described in [8]. The paper [9] investigates open source web service testing tools wildly for lack of parameters such as response time, number of data processed in terms of bytes and throughput which are then added to it through the modification of the tool.

The paper [10] presents a comparison made between selenium and other tools to find the best tool and on the basis of the results; a case study is presented to explore the performance of the selenium. An approach is used to analyze web application services and to report test results has been depicted in [11]. The approach uses Genetic Algorithm to generate Virtual Users which act as workloads. The generated workloads are applied to the System under Test for measuring various important performance indicators. The generated test reports help testers to measure performance of web application services under different conditions. 
Paper [12] describes a set of software tools to support the testing of web based applications. The tool set covers application model extraction, test execution automation, and test design automation. In addition, a graph based application model is also presented to model the behavior of web based applications. With the graphic presentation, several traditional software testing techniques are extended to test web based applications.

The paper [13] proposes the idea of modeling infrastructures as a general solution to the problems and describes a particular infrastructure that they have developed. It creates EMOD a tool for modeling the performance of e-commerce sites. It shows how EMOD can be used to model a particular site, and validate its predictions. An automatic conformance testing tool with timing constraints from a formal specification Timed Extended Finite State Machine of web services composition has been implemented in [14] by an online testing algorithm. This algorithm combines simultaneously the idea of test execution and debug to generate and simultaneously execute the test cases. The paper [15] describes the tool WSDLTest for automatic testing of web services. The tool can be used for testing of web services for which WSDL 1.1 or WSDL 2.0 documents are available.

The e-commerce site's privacy policy and security issue are examined by paper [17] to analyze user's personal data and security vulnerabilities. This survey study leverages penetration testing tools to collect data. Where, vulnerability issues of e-commerce sites have been experimented by [18] applying both technical and non-technical strategies. The study finds that vulnerabilities are exists in most of the e-commerce sites which causing unsecure for customers to trust their private data. The research [19] demonstrated the website's usability and accessibility issues for Nigerian banks websites. The study applied both manual and automated approach to evaluate the accessibility and usability issues and check the websites standard as defined by Web Accessibility Initiate.

\section{Empirical Data Collection}

E-commerce sites handles large number of users concurrently. As the number of users increasing day-by-day, the performance of the sites plays significant role to keep the user satisfied and thus keep site trends in a good shape. For this purpose, web application performance data collection system made automate in this proposed web based framework. Where three reputed web performance analyzer has been incorporated to collect and compare the ecommerce site data. The details regarding proposed e-commerce website performance comparing framework is presented in the following sections and depicted in Fig. 1.

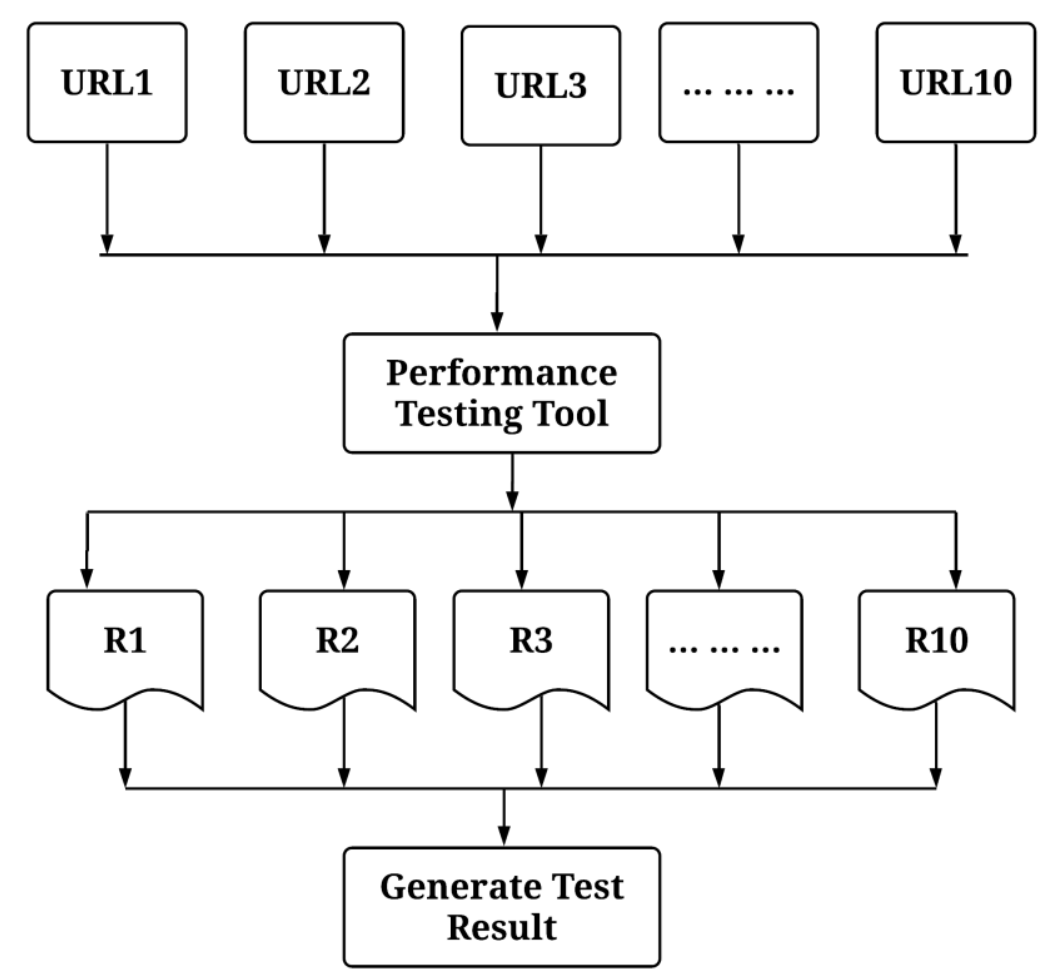

Fig. 1. Overview of the proposed method

The Fig. 1 illustrated the overview of the proposed system where a registered user can test the performance of any web application by providing the urls of the sites like url1, url2, ..., url10. The framework takes the urls as input and produce the test results of each website as R1, R2,.., R10. The system collects the performance records for the given 
url using three different tools which are (i) PageSpeed (ii) Insights, (iii) Webpage test. The results are stored to the database for further use and analysis. The developed web application creates a graphical comparison representation among all the sites based on considered performance parameters such as Load time, First Byte, Start Render, etc. using the stored results. The system has option to save the comparison results by the registered users.

\subsection{Data Scanning Tool Selection}

The developed framework incorporated three web performance analyzer to collect website performance data. These tools work as the fundamental tool of this web framework. The tools are selected based on their availability, functional performance, stability, etc. The overview of the experimented tools is given below.

- Webpage Test: This is one of the most popular and open source web performances testing tool. User can test a site considering different locations and browsers using this scanner.

- PageSpeed Insights: This is a reliable web performance analyzing to scan user experience for a given site accurately. For a web page, this tool generates performance results for both mobile and desktop devices. This is also a free tool.

- Gtmetrix: This is used to analyze the performance of a web page with a single click for different location, browsers, connection speed and more. The basic usage of this tool is free to test and monitor web pages' performance.

\subsection{Proposed Framework Development}

The system contains two modules named as frontend and backend. Both the module is developed with PHP, MySQL, CSS, HTML. The user will interact with frontend module to collection website performance records where user will register first with their details. The registration system requires username, user id, email, password, security question, security answer.

After getting registered with the framework, a user can scan web performance records of web applications by providing the respective website urls. The proposed framework will scan the given sites automatically using the considered three tools (i) PageSpeed (ii) Insights, (iii) Webpage test.

The control of the proposed system will remain under the admin user. Upon completion of performance testing, this system can compare and save the test result for a particular website. In addition, the application will generate a pdf file consisting of detail report for further use.

\section{Findings and Data Analysis}

The collected data has been analyzed and compared to identify the significant problems in the e-commerce sites. These results can help to mitigate the problem in the site in the next version release. For this empirical analysis, top ten e-commerce sites of Bangladesh has been considered to scan the data using this web based framework. All the discussed results are collected and analyzed using the proposed web based framework.

\subsection{Environment Setup}

For this empirical data collection following software and hardware has been used. HTML, CSS, JavaScript, PHP, MySQL, Fpdf17, XAMPP and windows OS has been used as software requirements. Whereas workstation consisting of intel core-i5, 50GB hard disk and 4 GB RAM is used to run the web application.

\subsection{Data Collection}

The data has been collected for nine parameters and plotted in Table 1, Table 2 and Table 3 for tools WebPage Test, PageSpeed Insight and Gtmetrix respectively. And the average results of these tools are shown in Table 4. The tables heading Site1, Site2, Site3, etc. are indicating the e-commerce site-1, site-2 site-3, etc. respectively. And the performance parameters named as (i) Load Time, (ii) First Byte, (iii) Start Render, (iv) First ContentFul Paint, (v) Speed Index, (vi) Largest Contentful Paint, (vii) Cumulative Layout Shift, (viii) Total Blocking Time and (ix) Time to Interactive are given in the row. The value represents in second and ceiled to two decimal points.

For collecting performance results using the proposed framework top e-commerce sites of Bangladesh has been selected according to a recent survey by an agency of Bangladesh which was published online. This paper is not mentioning the name of the sites because of preserving the privacy issue. 
Table 1. Performance Results for WebPage Test

\begin{tabular}{|l|l|l|l|l|l|l|l|l|l|l|l|}
\hline Sl. & \multicolumn{1}{|c|}{ Parameters } & Site1 & Site2 & Site3 & Site4 & Site5 & Site6 & Site7 & Site8 & Site9 & Site10 \\
\hline 1 & Load Time & 9.47 & 20.58 & 10.55 & 8.93 & 16.05 & 9.89 & 3.85 & 14.81 & 13.64 & 20.05 \\
\hline 2 & First Byte & 1.34 & 2.15 & 1.57 & 1.08 & 1.92 & 1.17 & 0.95 & 12.38 & 1.13 & 2.14 \\
\hline 3 & Start Render & 2.30 & 3.00 & 3.00 & 1.90 & 4.00 & 2.50 & 1.40 & 14.00 & 2.30 & 4.60 \\
\hline 4 & First Contentful Paint & 2.27 & 2.99 & 3.02 & 1.93 & 4.04 & 2.45 & 1.41 & 13.97 & 2.29 & 4.63 \\
\hline 5 & Speed Index & 3.52 & 14.29 & 3.33 & 2.96 & 7.74 & 3.96 & 1.66 & 14.06 & 8.58 & 12.39 \\
\hline 6 & Largest Contentful Paint & 6.64 & 3.89 & 3.45 & 4.23 & 7.41 & 5.97 & 1.56 & 14.24 & 7.56 & 15.64 \\
\hline 7 & Cumulative Layout Shift & 0.06 & 0.06 & 0.04 & 0.05 & 0.30 & 0.14 & 0.36 & 0.62 & 0.14 & 0.15 \\
\hline 8 & Total Blocking time & 0.14 & 1.03 & 0.61 & 0.00 & 0.87 & 0.55 & 0.00 & 0.00 & 1.23 & 0.31 \\
\hline 9 & Time to Interactive & 7.41 & 16.73 & 6.90 & 3.82 & 15.70 & 8.85 & 3.38 & 14.81 & 11.46 & 12.87 \\
\hline \multicolumn{2}{r}{ Average } & 3.68 & 7.12 & 3.61 & 2.77 & 6.45 & 3.95 & 1.62 & 10.99 & 5.37 & 8.09 \\
\hline
\end{tabular}

As stated in Table 1, WebPage test tool scanned for nine parameters and it shows the values for all. The parameter 'load time' shows 20.58 as maximum value for site2, which indicates the site needs more load time than others. Where, the site 7 records 3.85 as a minimum load time. For 'first byte' parameter it is lowest and highest value is 0.95 (site7) and 12.38 (site8) respectively. Considering average value for all the parameters, best and worst records are shown by site7 (1.62) and site8 (10.99) respectively. The tool shows 'total blocking time' parameter value as 0.00 for site4, site7 and site8.

Table 2. Performance Results for PageSpeed Insight

\begin{tabular}{|c|c|c|c|c|c|c|c|c|c|c|c|}
\hline Sl. & Parameters & Site1 & Site2 & Site3 & Site4 & Site5 & Site6 & Site7 & Site8 & Site9 & Site10 \\
\hline 1 & Load Time & - & - & - & - & - & - & - & - & - & - \\
\hline 2 & First Byte & - & - & - & - & - & - & - & - & - & - \\
\hline 3 & Start Render & - & - & - & - & - & - & - & - & - & - \\
\hline 4 & First Contentful Paint & 2.60 & 3.40 & 1.90 & 3.30 & 3.60 & 3.90 & 2.40 & 5.10 & 3.00 & 6.10 \\
\hline 5 & Speed Index & 9.30 & 12.90 & 6.10 & 4.10 & 10.20 & 11.90 & 13.70 & 14.40 & 16.20 & 8.90 \\
\hline 6 & Largest Contentful Paint & 14.9 & 9.00 & 2.90 & 4.10 & 8.80 & 17.30 & 2.80 & 5.40 & 14.80 & 7.60 \\
\hline 7 & Cumulative Layout Shift & 0.44 & 0.25 & 0.07 & 0.50 & 0.05 & 0.01 & 0.49 & 0.00 & 0.88 & 0.05 \\
\hline 8 & Total Blocking time & 1.32 & 11.05 & 2.84 & 0.22 & 2.08 & 1.55 & 0.07 & 0.45 & 2.18 & 0.16 \\
\hline 9 & Time to Interactive & 16.60 & 24.10 & 13.70 & 7.30 & 19.30 & 16.90 & 5.60 & 8.00 & 14.80 & 21.00 \\
\hline & Average & 7.53 & 10.12 & 4.59 & 3.25 & 7.34 & 8.60 & 4.18 & 5.56 & 8.64 & 7.30 \\
\hline
\end{tabular}

According to Table 2, the PageSpeed Insight scanner can collect records for (i) First Contentful Paint, (ii) Speed Index, (iii) Largest Contentful Paint, (iv) Cumulative Layout Shift, (v) Total Blocking Time and (v) Time to Interactive parameters. However, it is unable to detect any records for remaining three parameters. Among the scanned results, site8 shows 0.00 for 'cumulative layout shift', which indicates either the tool can not detect any record or the site consumes 0 second for this parameter. Where, the site 2 records 24.10 for 'time to interactive' parameter, so this site will take more time to be interactive compared to other experimented sites. The average minimum and maximum values are 3.25 (site4) and 10.12 (site2) respectively.

Table 3. Performance Results for Gtmetrix

\begin{tabular}{|c|c|c|c|c|c|c|c|c|c|c|c|}
\hline Sl. & Parameters & Site1 & Site2 & Site3 & Site4 & Site5 & Site6 & Site7 & Site8 & Site9 & Site10 \\
\hline 1 & Load Time & 7.90 & 13.20 & 6.90 & 7.00 & 10.80 & 8.70 & 4.00 & 13.10 & 13.40 & 15.50 \\
\hline 2 & First Byte & 1.20 & 2.00 & 1.10 & 0.10 & 1.30 & 1.50 & 1.30 & 11.30 & 1.30 & 1.20 \\
\hline 3 & Start Render & 1.50 & 2.10 & 1.70 & 1.70 & 2.80 & 2.70 & 1.70 & 12.20 & 2.30 & 2.90 \\
\hline 4 & First Contentful Paint & 1.50 & 2.20 & 1.70 & 1.70 & 2.80 & 2.70 & 1.70 & 12.20 & 2.30 & 2.90 \\
\hline 5 & Speed Index & 2.40 & 6.50 & 1.90 & 2.60 & 6.30 & 4.10 & 3.80 & 12.40 & 5.90 & 7.40 \\
\hline 6 & Largest Contentful Paint & 2.90 & 12.80 & 2.00 & 3.00 & 7.10 & 5.30 & 2.00 & 12.30 & 13.40 & 9.70 \\
\hline 7 & Cumulative Layout Shift & 0.09 & 0.07 & 0.00 & 0.11 & 0.50 & 0.01 & 0.48 & 0.57 & 0.61 & 0.25 \\
\hline 8 & Total Blocking time & 0.11 & 0.43 & 0.79 & 0.08 & 1.10 & 0.56 & 0.02 & 0.00 & 0.88 & 0.44 \\
\hline 9 & Time to Interactive & 2.00 & 7.10 & 5.00 & 3.90 & 9.70 & 3.50 & 2.00 & 12.20 & 9.90 & 8.00 \\
\hline & Average & 2.18 & 5.16 & 2.34 & 2.24 & 4.71 & 3.23 & 1.89 & 9.56 & 5.55 & 5.37 \\
\hline
\end{tabular}

As stated in Table 3, the site 3 and site 8 shows 0.00 second in case of 'cumulative layout shift' and 'total blocking time' respectively. The tool records highest and lowest 'load time' as 15.50 and 4.00 seconds for the site 10 and site7 respectively. Even though, the site site 8 shows 0.00 second for 'total blocking time', it records maximum time for (i) first byte, (ii) Start Render, (iii) First Contentful Paint, (iv) Speed Index, (v) Largest Contentful Paint and (vi) Time to Interactive parameters. The lowest and highest average performance values are 1.89 (site7) and 9.56 (site8) seconds respectively. 
Table 4. Average of WebPage Test, PageSpeed Insight and Gtmetrix

\begin{tabular}{|c|c|c|c|c|c|c|c|c|c|c|c|}
\hline SI. & Parameters & Site1 & Site2 & Site3 & Site4 & Site5 & Site6 & Site7 & Site8 & Site9 & Site10 \\
\hline 1 & Load Time & 8.69 & 16.89 & 8.73 & 7.97 & 13.43 & 9.30 & 3.93 & 13.96 & 13.52 & 17.78 \\
\hline 2 & First Byte & 1.27 & 2.06 & 1.34 & 0.59 & 1.61 & 1.34 & 1.13 & 11.84 & 1.215 & 1.67 \\
\hline 3 & Start Render & 1.90 & 2.55 & 2.35 & 1.80 & 3.40 & 2.60 & 1.55 & 13.10 & 2.30 & 3.75 \\
\hline 4 & First Contentful Paint & 2.12 & 2.86 & 2.21 & 2.31 & 3.48 & 3.02 & 1.84 & 10.42 & 2.53 & 4.54 \\
\hline 5 & Speed Index & 5.07 & 11.23 & 3.78 & 3.22 & 8.08 & 6.65 & 6.39 & 13.62 & 10.23 & 9.56 \\
\hline 6 & Largest Contentful Paint & 8.15 & 8.56 & 2.78 & 3.78 & 7.77 & 9.52 & 2.12 & 10.65 & 11.92 & 10.98 \\
\hline 7 & Cumulative Layout Shift & 0.20 & 0.13 & 0.04 & 0.22 & 0.28 & 0.05 & 0.44 & 0.397 & 0.54 & 0.15 \\
\hline 8 & Total Blocking time & 0.52 & 4.17 & 1.41 & 0.10 & 1.35 & 0.89 & 0.03 & 0.15 & 1.43 & 0.30 \\
\hline 9 & Time to Interactive & 8.67 & 15.98 & 8.53 & 5.01 & 14.9 & 9.75 & 3.66 & 11.67 & 12.05 & 13.96 \\
\hline & Average & 4.07 & 7.16 & 3.46 & 2.78 & 6.03 & 4.80 & 2.34 & 9.53 & 6.19 & 6.97 \\
\hline
\end{tabular}

The measured average performance records for each parameter has been plotted in Table 4. The average value has been calculated using considering the three tools scanning output. According to this table, the lowest value is 0.03 for 'Total Blocking Time' by site7. And highest value is 17.78 seconds recorded by site10 for 'Load Time' parameter. The three tools' lowest and highest average performance is 2.34 and 9.53 seconds for site 7 and site 8 respectively.

Beside individual tool's results analysis, the proposed framework generates average results as Table 4 to compare each parameter value for ten sites. As the Pagespeed tool could not collect data for 'Load Time', 'First Byte' and 'Start Render' parameters, the average is calculated using the records of Webpage Test and Gtmetrix tools. Using Table 4, a user can easily find out the major performance difference among the sites and can take necessary action.

\section{Conclusion}

This paper presents a web based framework for performance analysis for any given website automatically. It can show the developers how a website can behave and respond during various situations. It will show them the speed and stability of their tested websites. To use this system, a user needs to register and provide the website's url for performance analysis of the given web application. The framework uses Webpage Test, PageSpeed Insights and Gtmetrix web performance tools to scan the given website url. The performance records have been collected for nine parameters such as Load Time, First Byte, Start Render, First Contentful Paint, Speed Index, Largest Contentful Paint, Cumulative Layout Shift, Total Blocking Time and Time to Interactive parameters. The individual scanner records as well as average of three scanners have been generated by the developed framework in order to analyze status of the performance of the sites. According to the average results, site7 scored lowest for 'Total Blocking Time' among the considered ten sites. The site10 scored 17.78 seconds as Load Time, which indicates that this site takes more loading time compare to others. At present, this web based application works only in the computer system. Development of mobile version as well as increase number of test limit will be the future research direction.

\section{References}

[1] Shakti Kundu," Web Testing: Tool, Challenges and Methods”, IJCSI International Journal of Computer Science Issues, Vol. 9, Issue 2, No 3, March 2012.

[2] Sherolwendy Anak Sualim, Noraniah Mohd Yassin, and Radziah Mohamad," Comparative Evaluation of Automated User Acceptance Testing Tool for Web Based Application", International Journal of Software Engineering and Technology, IJSET Vol. 2, No. 2 (2016)

[3] G. Ruffo, R. Schifanella, M. Sereno and R. Politi ,"WALTy: A User Behavior Tailored Tool for Evaluating Web Application Performance", Proceedings of the Third IEEE International Symposium on Network Computing and Applications (NCA'04).

[4] Mahnaz Shams, Diwakar Krishnamurthy, and Behrouz Far," A Model-Based Approach for Testing the Performance of Web Applications", Proceedings of the Third International Workshop on Software Quality Assurance (SOQUA'06)

[5] Shikha Raina and Arun Prakash Agarwal," An Automated Tool for Regression Testing in Web Applications", ACM SIGSOFT Software Engineering Notes Volume 38, Issue 4.

[6] Fredrik Abbors, Tanwir Ahmad, Dragos, Trus,can, and Ivan Porres," MBPeT: A Model-Based Performance Testing Tool”, 4th International Conference on Advances in System Testing and Validation Lifecycle, November 2012

[7] Zhen Ming Jiang, Ahmed E. Hassan ,and Gilbert Hamann and Parminder Flora," Automated Performance Analysis of Load Tests", 2009 IEEE International Conference on Software Maintenance, 30 October 2009

[8] Renu Patil, and Rohini Temkar," Intelligent Testing Tool: Selenium Web Driver", International Research Journal of Engineering and Technology (IRJET), Volume: 04 Issue: 06, June -2017.

[9] Tanuj Wala , and Aman Kumar Sharma," Improvised Software Testing Tool”, , International Journal of Computer Science and Mobile Computing, Vol.3 Issue.9, September- 2014, page 573-581

[10] Hafsah Mahmood, and Mehreen Sirshar," A Case Study of Web Based Application by Analyzing Performance of a Testing Tool", International Journal of Education and Management Engineering (IJEME), 51-58 page, July 2017

[11] Ms.B.Shyaamini, and Dr.M.Senthilkumar," A Novel Approach for Performance Testing On Web Application Services", International Journal of Applied Engineering Research, Volume 10, Number 18 (2015) 
[12] Ji-Tzay Yang, Jiun-Long Huang, and Feng-Jian Wang," A Tool Set to Support Web Application Testing”, International Computer Symposium (ICS), October 1998

[13] Jonathan C. Hardwick, Efstathios Papaefstathiou, and David Guimbellot," Modeling the Performance of E-Commerce Sites", Proceedings of the 27th International Conference of the Computer Measurement Group, January 2001

[14] Tien-Dung Cao, Patrick Felix, and Richard Castanet," WSOTF: An Automatic Testing Tool for Web Services Composition", 2010 Fifth International Conference on Internet and Web Applications and Services, 2010 IEEE

[15] Ilona Bluemke, Michał Kurek, and Małgorzata Purwin," Proceedings of the 2014 Federated Conference on Computer Science and Information Systems", Vol. 2, pp. 1553-1558.

[16] Mahfida Amjad, Md. Tutul Hossain, Rakib Hassan, Md. Abdur Rahman, " Web Application Performance Analysis of ECommerce Sites in Bangladesh: An Empirical Study", International Journal of Information Engineering and Electronic Business (IJIEEB), Vol.13, No.2, pp. 47-54, 2021. DOI: 10.5815/ijieeb.2021.02.04.

[17] Issah Baako, Sayibu Umar, Prosper Gidisu, "Privacy and Security Concerns in Electronic Commerce Websites in Ghana: A Survey Study", International Journal of Computer Network and Information Security(IJCNIS), Vol.11, No.10, pp.19-25, 2019. DOI: $10.5815 /$ ijcnis.2019.10.03.

[18] Issah Baako, Sayibu Umar, "An Integrated Vulnerability Assessment of Electronic Commerce Websites", International Journal of Information Engineering and Electronic Business(IJIEEB), Vol.12, No.5, pp. 24-32, 2020. DOI: 10.5815/ijieeb.2020.05.03.

[19] Ishaq O. Oyefolahan, Aishat A. Sule, Solomon A. Adepoju, Faiza Babakano, "Keeping with the Global Trends: An Evaluation of Accessibility and Usability of Nigerian Banks Websites.", International Journal of Information Engineering and Electronic Business(IJIEEB), Vol.11, No.2, pp. 44-53, 2019. DOI: 10.5815/ijieeb.2019.02.06.

\section{Authors' Profiles}

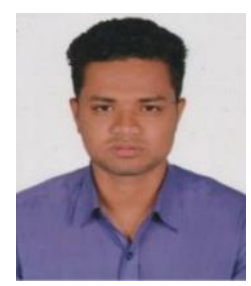

Md. Tutul Hossain was born in 1997 In Narail, Dhaka, Bangladesh. He has completed his B.Sc in ComputerScience and Engineering from Stamford University Bangladesh in 2020. Currently he is working on WordPress Developer at Buy Perfume in Bangladesh (BPIB).

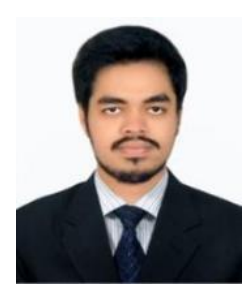

Rakib Hassan was born in 1997 in Dhaka, Bangladesh. He has completed his B.Sc degree in Stamford University.

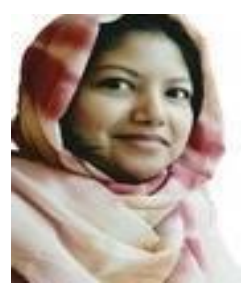

Mahfida Amjad was born in 1985 in Dhaka, Bangladesh. She has completed her Master degree in Information Technology from Institute of Information Technology from University of Dhaka in 2009. And she completed B.Sc. in Computer Science \& Engineering from Manarat International University in 2007. She is a faculty member of Computer Science and Engineering (CSE) Department of Stamford University Bangladesh. She has devoted herself in teaching profession since 2012. Her research area is wireless communication network, human computer interaction and software engineering. She has published a number of research papers in various international journals and conferences.

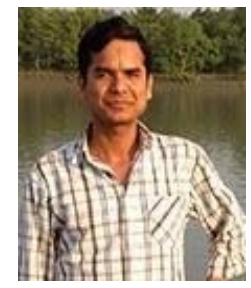

Md. Abdur Rahman received his BSc in Information Technology from Visva Bharati University, India in 2004 He has completed his Post Graduate Diploma and Master in Information Technology from University of Dhaka, Bangladesh, in 2008 and 2009 respectively. He is a Senior Computer Scientist at the Centre for Advanced Research in Sciences, University of Dhaka. His major research interest includes text analytics, application of machine and deep learning in software engineering and natural language processing. He has published a number of research papers in various international journals and conferences. 
How to cite this paper: Md. Tutul Hossain, Rakib Hassan, Mahfida Amjad, Md. Abdur Rahman, " Web Performance Analysis: An Empirical Analysis of E-Commerce Sites in Bangladesh", International Journal of Information Engineering and Electronic Business(IJIEEB), Vol.13, No.4, pp. 47-54, 2021. DOI: 10.5815/ijieeb.2021.04.04 